\title{
RFID zur Absicherung von Prozessen und Erlösen im OP
}

In den letzten zehn Jahren sind die Fallzahlen gestiegen und mit ihnen die Anzahl der Operationen (Steigerung um 25 Prozent). Nun gilt es, die damit verbundenen administrativen Prozesse optimal und mit geringem Zusatzaufwand zu integrieren.

Bis zu 60 Prozent der Behandlungskosten entstehen am Tag der Operation. Deshalb ist es von größter Bedeutung, dass die Operationssäle durch gute Steuerung und Abläufe optimal ausgelastet sind. Mit der Zunahme von Operationen stieg u.a. in diesem Kontext der Anteil von Implantaten und Einmalsterilgut. Vor dem Hintergrund gesetzlicher Vorgaben muss dieses Einmalsterilgut zum Teil zwingend dokumentiert werden (z. B. UDI) und es sollte aufgrund ökonomischer Überlegungen auch verbrauchswertabhängig dokumentiert werden. In Deutschland verbindet man hiermit einen Abgleich mit der InEK-Kalkulation, welche seitens der Krankenhäuser zwar nur auf indirektem Wege langfristig beeinflusst werden kann, deren Grenzkostensätze mit Blick auf die Vermeidung von Verlusten jedoch unbedingt einzuhalten sind. In Ländern mit direkt verbrauchsbezogener Abrechnung (z. B. in den Niederlanden) ist das Festhalten des Verbrauchs direkt an den Erlös gebunden und damit obligatorisch. Unabhängig von den Beweggründen ist es für ein Operationsteam wichtig, dass die Dokumentation mit möglichst wenig Aufwand erfolgt.

\section{Viel Arbeitszeit für fachfremde Tätigkeiten}

Laut einer Reihe von Studien widmen sich Mitarbeiter der Pflege immer noch einen Großteil ihrer Arbeitszeit logistischen Aufgaben, welche insbesondere durch Annahme, Erfassung und Inventuraufgaben entlang der Supply Chain entstehen. In diesem Kontext wurden in den letzten Jahren Lösungen diskutiert, die von der Auslieferung beim Hersteller bis zur Ver-

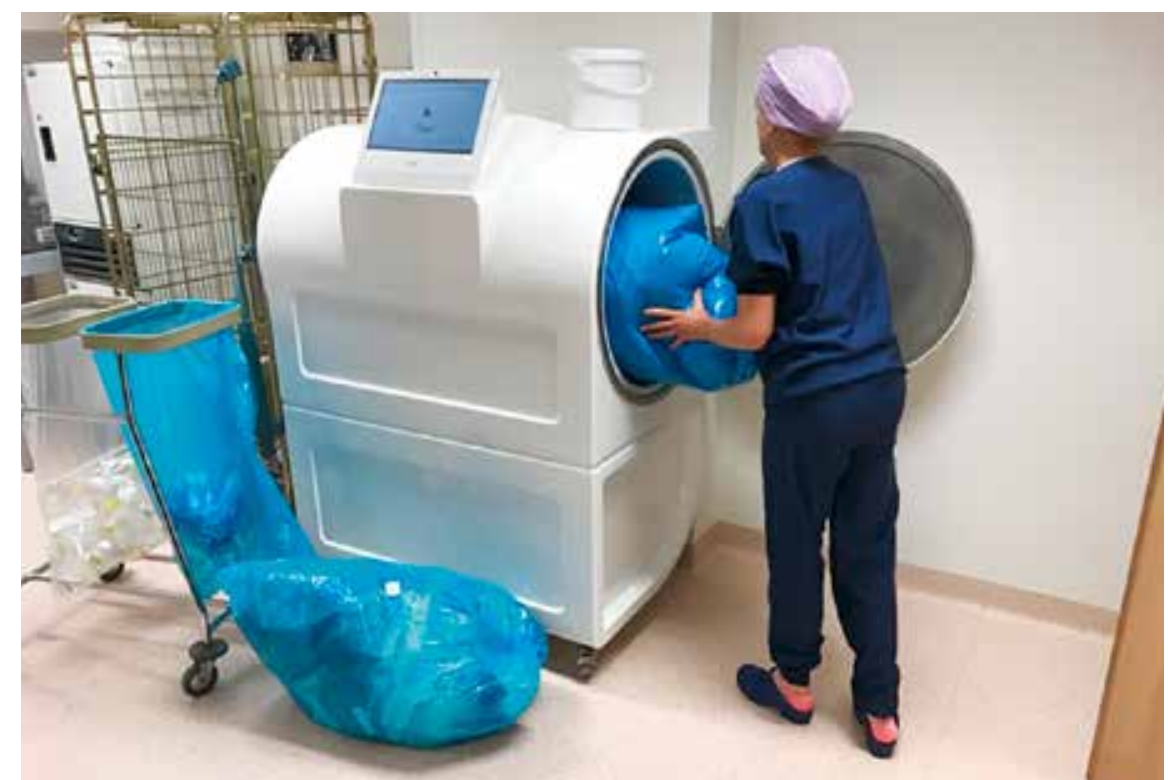

Befüllen des als Drehtrommel ausgeführten RFID-Readers. Foto: CWZ, Nijmegen, Niederlande

Bis zu 60 Prozent der Behandlungskosten entstehen am Tag der OP.

wendung im Operationssaal effiziente, transparente und dokumentierte Prozesse sicherstellen und idealerweise automatisiert abbilden.

\section{Systemlösung zur Automatisierung administrativer Prozesse}

Eine zukunftweisende Entwicklung, welche die genannten Anforderungen erfüllt, stellt z. B. das System Resolution der Fa. Johnson \& Johnson dar. Hierbei werden Sterilgutverpackungen mit einem RFID-Chip nach dem Peelen (Auspacken) im OP-Saal in einen Plastiksack gewor- fen. Dieser Vorgang entspricht 1:1 dem üblichen Procedere im OP, wobei ein Abscannen oder Eintippen ausgewählter Artikelangaben entfällt. Nach OP-Ende wird der Sack, in dem sich neben den leeren Verpackungen ein RFID-Tag mit der ID des Patienten befindet, in einen als Drehtrommel ausgeführten Reader gelegt. Durch die Rotation der Trommel beim Auslesevorgang ist eine Erkennung aller im Sack befindlichen Verpackungen sichergestellt (ein wichtiger Punkt, der bei Verwendung statischer Reader problematisch ist) und die Lesezeit beläuft sich auf nur wenige Sekunden. Abschließend wird der Sack entsorgt.

Die Integration der Lösung in die Supply Chain bis hin zur Dokumentation 


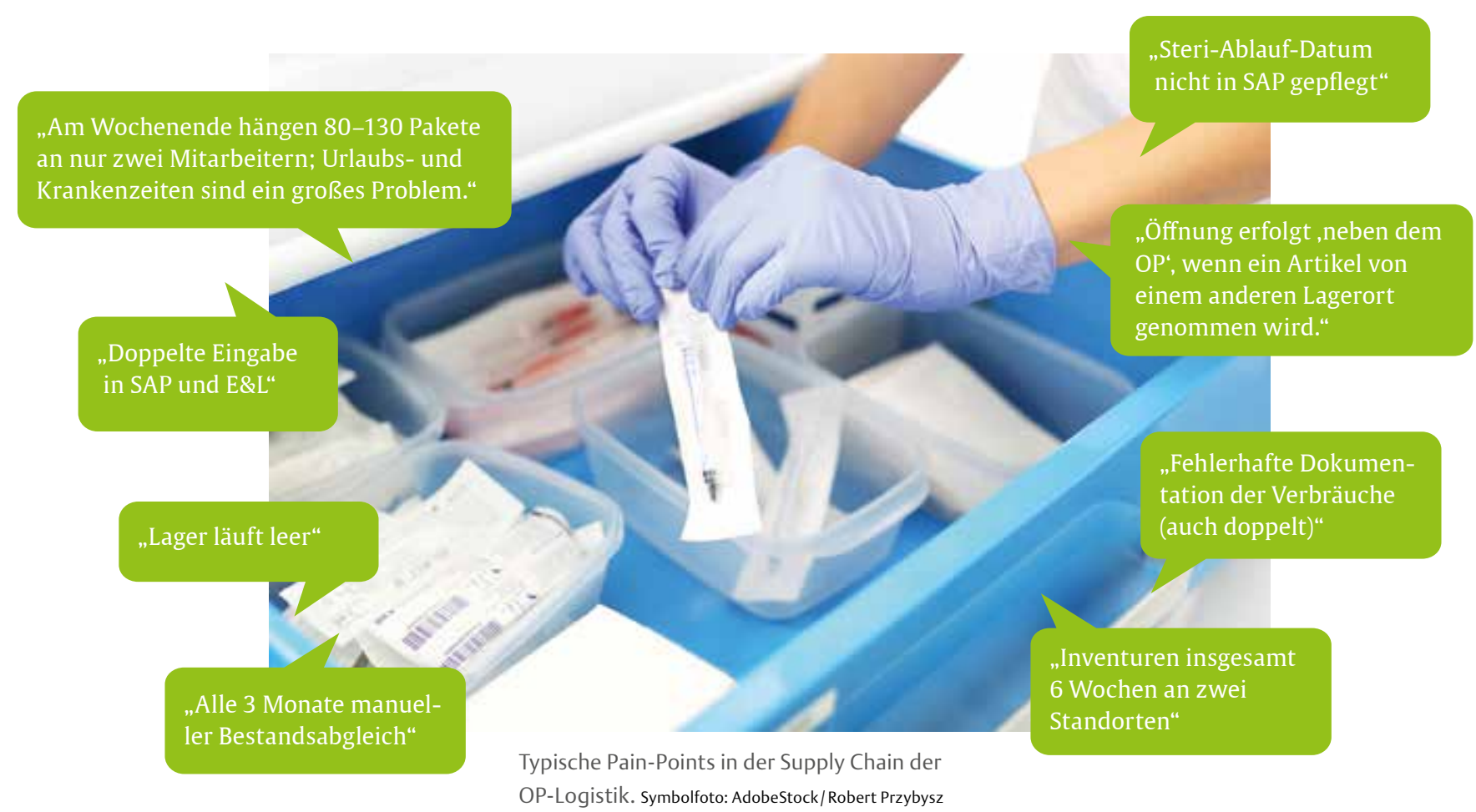

ermöglicht eine medienbruchfreie Auftragsbearbeitung von der Bedarfsanforderung über Einkauf, Wareneingang und Lagerverwaltung sowie eine elektronische Verknüpfung von verbrauchten Produkten bzw. Materialien zu Patienten und Eingriffen. Damit wiederum können konkrete Erlöse in der InEK-Kalkulationsmatrix adressiert und abgeglichen werden.

\section{Erfahrungen in 3 Ländern auf 2 Konti- nenten}

Nach dem Rollout in Australien wurde Resolution Schritt für Schritt optimiert und ist mittlerweile technisch ausgereift. Seit ca. zwei Jahren befindet sich das System auch in Krankenhäusern in Italien und den Niederlanden im Einsatz. Das Tagging, also das Aufbringen des RFIDChips auf die Verpackung erfolgt hierbei je nach vorhandener Infrastruktur und Differenzierung von Lagerartikeln und Durchläufern entweder im Zentrallager und/oder im OP-Lager. Aktuell wird die Machbarkeit in Zusammenarbeit mit der Einkaufsgemeinschaft AGKAMED geprüft. Die AGKALogistics mit dem Zentrallager der AGKAMED bietet den hier angeschlossenen Krankenhäusern an, das Tagging zu übernehmen.
„Der Erfolg ergibt sich aus der Arbeitszeitersparnis und der Dokumentationsqualität“, so Dr. Oliver Gründel, Geschäftsführer der AGKAMED. Er möchte „die Logistik an die Bestellfunktion heranführen und dazu ist das System absolut ideal, da ein Ausbuchen eines Artikels einen Nachbestellprozess auslöst“. Dies wird durch eine in den Niederlanden erprobte Datenintegrationslösung sichergestellt, die über standardisierte Schnittstellen Resolution mit dem Krankenhaus-ERPSystem verbindet.

\section{Qualitative Bewertung}

Zu einer positiven Bewertung kommt auch das Institut für Healthcare Management (IHCM) an der Hochschule Niederrhein in Krefeld. In einer Value Study verglichen die Wissenschaftler die Ist-Situation (Daten und Prozesse) von 3 Maximalversorgern und 2 Krankenhäusern der Grund- und Regelversorgung aus Deutschland mit denen zweier Kliniken in Italien sowie einem Krankenhaus in den Niederlanden, in denen sich Resolution seit mehr als einem Jahr im Regelbetrieb befindet. Aufgrund der vor und nach der Einführung von Resolution geführten Statistiken bzgl. häufig vorkommender Fehler (z.B. in der
99 Es ist mit Kosteneinsparungen von rund 20000 Euro pro Jahr bei 15000 Operationen und durchschnittlich 2,5 Artikeln je Operation zu rechnen, wobei sich die Systemlösung dann in ca. 3 Jahren amortisiert.“

Prof. Dr. Hubert Otten, geschäftsführender Institutsleiter am IHCM 


\section{Amortisationszeit bei zwei mit RFID-Chips getagten Artikeln je Operation}

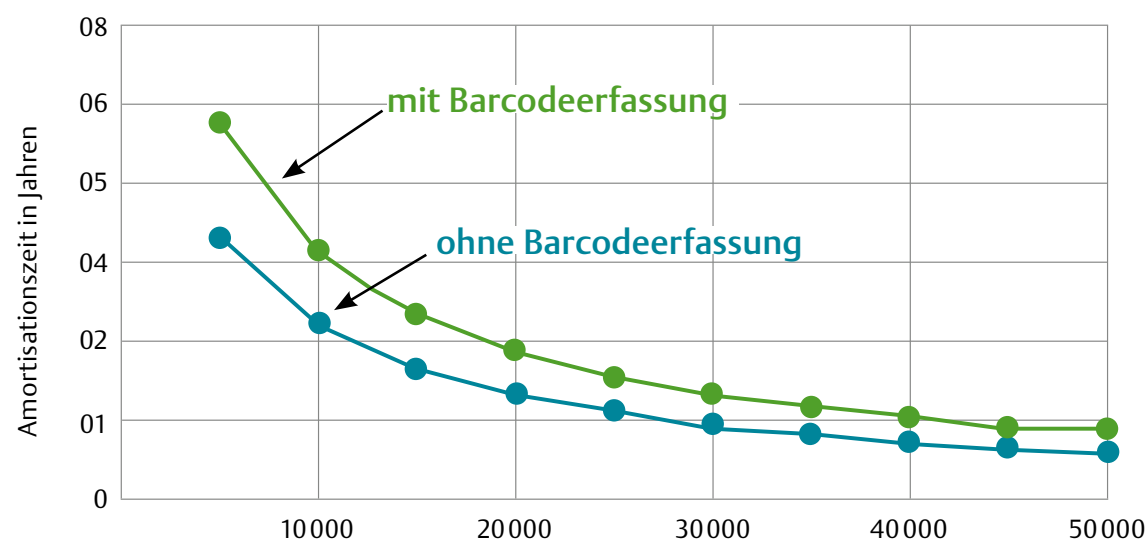

Anzahl der Operationen pro Jahr

Quelle: Hubert Otten

Nutzenpotenziale

von RFID

schnellere und qualitätsgesicherte OP-Dokumentation

OP-/Patienten-/Fallzuordnung

$\checkmark$ schnellere Inventur

beschleunigter und verbesserter (durch Bestellmengenoptimierung) Bestell- und Wareneingangsprozess

• verringerte Risiken

• Patient: generelle Erhöhung der Patientensicherheit und Chance zur Verweildauerreduzierung und Verringerung der Komplexitätsrate im Rahmen innovativen Medizincontrollings

- Krankenhaus: Sicherstellung ausreichender fallbezogener Erlöse und Absicherung von kostenintensiven Identifikationsfehlern

- OP-Pflege: entspannte Arbeitsatmosphäre mit geringerem Stressfaktor und reduzierter Fehlerwahrscheinlichkeit
Dokumentation) und Defizite (z.B. Überlagerung von Sterilgut), die im Weiteren in mehreren hiervon unabhängigen wissenschaftlichen Studien im Themenkomplex RFID im Gesundheitswesen und in der Pharmazie auf Plausibiltität geprüft und bestätigt wurden, ergaben sich diverse Nutzenpotenziale (s. Kasten).

\section{Quantifizierbarer Nutzen}

Auf Grundlage der durchgeführten Erhebungen konnten neben den qualitativen Potenzialen Kennzahlen für eine Abschätzung des quantitativen Nutzens entwickelt und näherungsweise berechnet werden. Hierbei wurden mit Blick auf die grundsätzlichen Unterschiede in vielen Krankenhäusern die Variante mit und ohne Barcodeerfassung im Ist-Zustand unterschieden. Unter Zugrundelegung von Prozessaufwand in Logistik und Dokumentation sowie bei konservativem Einbezug von gemessenen Fehlzuständen in den Pilothäusern ist mit „Kosteneinsparungen von rund 20000 Euro pro Jahr bei 15000 Operationen und durchschnittlich 2,5 Artikeln je Operation zu rechnen, wobei sich die Systemlösung dann in ca. 3 Jahren amortisiert“, so Prof. Dr. Hubert Otten, geschäftsführender Institutsleiter und verantwortlich für den Bereich Technische Systeme, Betriebsorganisaiton und Logistik am IHCM (Abbildung 3).

\section{Einrichtung eines Demonstrations- zentrums}

Die positive Resonanz hat Johnson \& Johnson veranlasst, in Norderstedt ein Demonstrationszentrum einzurichten, das Interessierten die Lösung am praktischen Beispiel veranschaulicht und in dem die bislang gemachten Erfahrungen mit Dr. Thomas Burger, (Senior Program Manager) und Sebastian Dahlem (Project Manager Value Added Services) diskutiert werden können. „Es ist überraschend, wie viel Optimierungspotential wir in detailierten Supply Chain Prozessanalysen aufdecken und in welchem Maße Handlungsbedarfe abgeleitet werden können" so Sebastian Dahlem.

Dem schließt sich Dr. Gründel von der AGKAMED an: „Die Krankenhäuser haben vor allem ein Personalproblem im Bereich der Funktionsstellen. Hier verringern administrative Aufgaben, die oft nicht gut organisiert, geschweige denn wie in anderen Branchen automatisiert sind, die Zeit, die eigentlich für die Patienten zur Verfügung stehen sollte. Deshalb ist das Thema besonders wichtig und innovativ für uns und unsere Häuser.“

Prof. Dr. Hubert Otten, Institutsleiter Institut für Datenmanagement und Datenvalidierung im Gesundheitswesen e.V., Leiter Competence Center eHealth und Professor für Technische Systeme, Betriebsorganisation und Logistik in Einrichtungen des Gesundheitswesens, Hochschule Niederrhein

Sebastian S. Dahlem, Project Manager bei Johnson \& Johnson Medical $\mathrm{GmbH}$

Andreas Petri, Wissenschaftlicher Mitarbeiter am Institut für Healthcare Management, Hochschule Niederrhein 